\title{
Measurement of Internuclear Distances by Switched Angle Spinning NMR
}

\author{
Andrew C. Kolbert," Philip J. Grandinetti, ${ }^{\dagger}$ Michael Baldwin, $\ddagger$ Stanley B. Prusiner, ${ }^{\ddagger}$ and \\ Alexander Pines \\ Material Sciences Division, Lawrence Berkeley Laboratory, and Department of Chemistry, \\ University of California, Berkeley, California 94720
}

Received: April 26, 1994; In Final Form: June 27, $1994^{\circ}$

\begin{abstract}
Dipolar switched angle spinning, an NMR technique due to Terao et al. [Terao, T.; Miura H.; Saika, A. J. Chem. Phys. 1986, 85, 3816], has been used to measure ${ }^{13} \mathrm{C}-{ }^{13} \mathrm{C}$ distances in the solid state. The experiment involves rotation of the sample at two different angles during different periods of a two-dimensional experiment. An evolution period with off-magic-angle spinning and chemical shift refocusing, followed by detection of the signal under magic angle spinning, yields scaled Pake patterns in $\omega_{1}$, correlated with their isotropic shifts in $\omega_{2}$, allowing the high-resolution measurement of dipolar couplings. We demonstrate this experiment on samples of doubly ${ }^{13} \mathrm{C}$ labeled zinc acetate and a 14 amino acid peptide, in which we show that under optimal conditions distances of up to $5 \AA$ may be measured.
\end{abstract}

\section{Introduction}

The measurement of interatomic distances with solid state nuclear magnetic resonance (NMR) has recently attracted a great deal of attention, ${ }^{1-5}$ largely because solid state NMR is one of the only methods of obtaining structural information on materials lacking long range order. Several techniques have been developed to extract distances between homonuclear coupled spin pairs. Yannoni et al. have developed nutation NMR which utilizes a strong radio-frequency (rf) field to average chemical shifts, while preserving the dipolar interaction, scaled by one-half.6,7 Griffin and co-workers have recently employed longitudinal magnetization exchange via rotational resonance to measure distances between ${ }^{13} \mathrm{C}$-labeled spin pairs of up to $7 \AA$ with a $0.5-\AA$ resolution. ${ }^{1-3,8,9}$ The magnetization transfer curves thus obtained are sensitive to the dipolar coupling and therefore the internuclear distance. Several methods involving rotor synchronized pulse sequences have recently been invented to reintroduce the homonuclear dipolar interaction during magic angle spinning. $5,10,11$ Terao and co-workers have previously investigated strong heteronuclear dipolar couplings by correlating magic angle spinning (MAS) spectra with static heteronuclear dipolar powder patterns, ${ }^{12}$ accomplished by spinning off the magic angle during the evolution period of a two-dimensional experiment, switched angle spinning (SAS). The spectra obtained along the $\omega_{1}$ axis are dependent only upon the dipolar coupling, allowing the straightforward extraction of the internuclear distance. As the dipolar couplings were large and involved proton spins, small rotor flip angles were used as well as proton multiple-pulse decoupling during the $t_{1}$ period. Recently, in our laboratory, instrumentation for dynamic angle spinning of quadrupolar nuclei has been developed which involves the rapid reorientation of the sample spinning axis by up to $63^{\circ} .13$ This has prompted us to investigate an extension of the method of Terao et al. ${ }^{12}$ to the measurement of weak homonuclear dipolar couplings which require large deviations from the magic angle in $\omega_{1}$ so as to avoid excessive scaling of the dipolar interaction. Tycko has recently reported a related application of switched angle spinning to the problem of multiple quantum filtering during MAS. ${ }^{14}$

\footnotetext{
† Present address: Department of Chemistry, Ohio State University, Columbus, $\mathrm{OH}$.

¥Department of Neurology, University of California, San Francisco, California.

- Abstract published in Advance ACS Abstracts, August 1, 1994
}

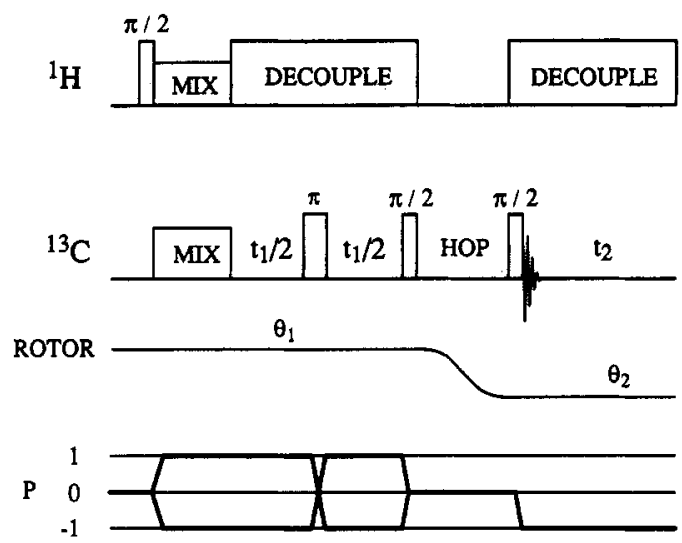

Figure 1. Pulse sequence for dipolar switched angle spinning NMR. The coherence transfer pathway is shown beneath.

\section{Results and Discussion}

The correlation of MAS spectra with dipolar patterns is implemented via the pulse sequence of Figure 1. Following crosspolarization, the coherence evolves for a time $t_{1}$ under the influence of the homonuclear dipolar interaction, the chemical shifts being refocused by a $\pi$ pulse at $t_{1} / 2$. The $\pi$ pulse is asynchronous with the sample rotation and will result in spinning sidebands spaced at one-half of the spinning speed in $\omega_{1}$, as shown previously. ${ }^{15-17}$ During this evolution period the rotor is spinning at $\theta_{1}$, usually $0^{\circ}$ or $90^{\circ}$, leading to a nonvanishing two-spin dipolar coupling, which varies as $P_{2}\left(\cos \theta_{1}\right)$. After the evolution period, the coherence is then stored along the $z$ axis with a $\pi / 2$ pulse, the rotor axis is hopped to $\theta_{2}$, typically the magic angle, the coherence is restored to the $x-y$ plane, and the signal is detected. Highpower proton decoupling is applied during the entire experiment, except during the axis hop period. Two-dimensional Fourier transformation of the resulting signal, acquired as a function of $t_{1}$ and $t_{2}$, yields a high-resolution MAS spectrum correlated with a two-spin dipolar pattern scaled by $P_{2}\left(\cos \theta_{1}\right)$. This is demonstrated by the 2D dipolar SAS spectrum of di-13 $\mathrm{C}$-labeled zinc acetate as shown in Figure 2. The spectrum was acquired with $\theta_{1}=90^{\circ}$ rather than $\theta_{1}=0^{\circ}$, leading to a scaling factor of 0.5 , due to the difficulty of performing ${ }^{1} \mathrm{H}$ decoupling with the sample spinning about the $z$ axis. For small dipolar couplings, spinning about the $z$ axis during the $t_{1}$ period may be desirable to overcome the scaling factor of $0.5 .^{18}$ Well-resolved dipolar patterns are observed along the $\omega_{1}$ axis, correlated with their MAS spectra along the $\omega_{2}$ axis. The dipolar spectra are distorted 


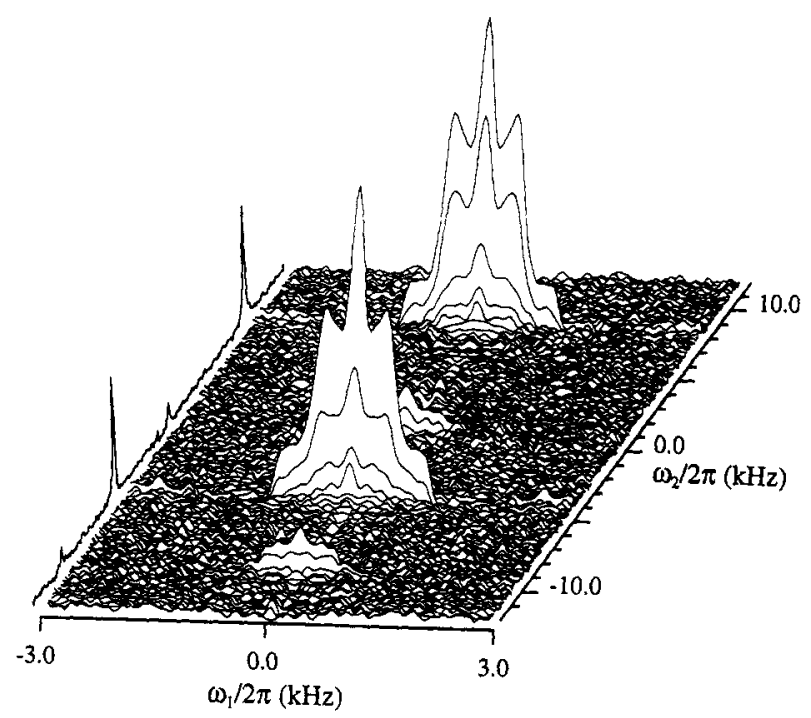

Figure 2. 2D Dipolar SAS spectrum of di- ${ }^{13} \mathrm{C}$-labeled $\mathrm{Zn}(\mathrm{Ac})_{2}$ diluted 1:10 in natural abundance $\mathrm{Zn}(\mathrm{Ac})_{2}$. A total of $64 t_{1}$ increments were acquired with 256 scans per FID and a recycle time of $1 \mathrm{~s}$. The dwell times were 41.7 and $100 \mu \mathrm{s}$ in $t_{2}$ and $t_{1}$, respectively, and the spinning speed was $5110 \mathrm{~Hz}$. The hop time was approximately $50 \mathrm{~ms}$, and the angle of the rotor with respect to the magnetic field was $90^{\circ}$ during $t_{1}$.

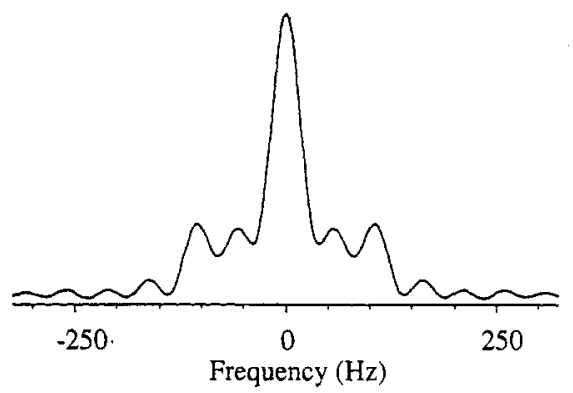

Figure 3. Slice taken from a 2D dipolar SAS spectrum parallel to the $\omega_{1}$ axis at a point corresponding to the $\mathrm{COO}^{-}$line center band in $\omega_{2}$. The rotor was at $\theta_{1}=52.7^{\circ}$ during $t_{1}$ and at the magic angle for detection. The scaling factor of the homonuclear dipolar coupling due to the offangle spinning during $t_{1}$ is approximately $5 \%$. Line narrowing of $-30 \mathrm{~Hz}$ has been applied to the FID in $\omega_{1}$, to enhace the singularities.

by an additional peak at zero frequency, presumably due to isolated single ${ }^{13} \mathrm{C}$ labels in the sample. The dipolar coupling of $b_{1 \mathrm{~s}}=$ $\gamma^{2} h / 2 \pi r^{3}=2.00 \mathrm{kHz}$, corresponding to a $1.5-\AA$ distance, may easily be measured from either the parallel or perpendicular edges. Figure 3 shows a slice extracted from a $2 \mathrm{D}$ spectrum acquired as in Figure 2, with the difference that $\theta_{1}=52.7^{\circ}, 2^{\circ}$ away from the magic angle, yielding a scaling factor of 0.05 . This appears to be close to the resolution limit for this experiment, and corresponds to a spin pair with a $4.2-\AA$ distance, were the experiment performed with $\theta_{1}=0^{\circ}$. The spectra obtained in this manner are sensitive only to the dipolar coupling between sites and are unaffected by rf inhomogeneity. 6,7 For dipolar couplings which are comparable to the chemical shift separation, second order effects may become important resulting in patterns that reflect the imperfect refocusing of the chemical shifts in $\omega_{1} .{ }^{19}$ For the examples reported here, the dipolar couplings are substantially smaller than the shift differences, and the Hamiltonian can be considered to be properly truncated at first order. In situations in which a nonideal pake pattern is observed, an exact calculation of the dipolar and two chemical shift tensor Hamiltonian is required to extract accurate distances from fits to the $\omega_{1}$ projections. ${ }^{19}$

When the homogeneous line width becomes comparable to the dipolar splittings in the static powder pattern, the distance can nolonger be measured directly from the singularities in $\omega_{1}$. Rather, the $\omega_{1}$ spectra must be compared with broadened simulated pake

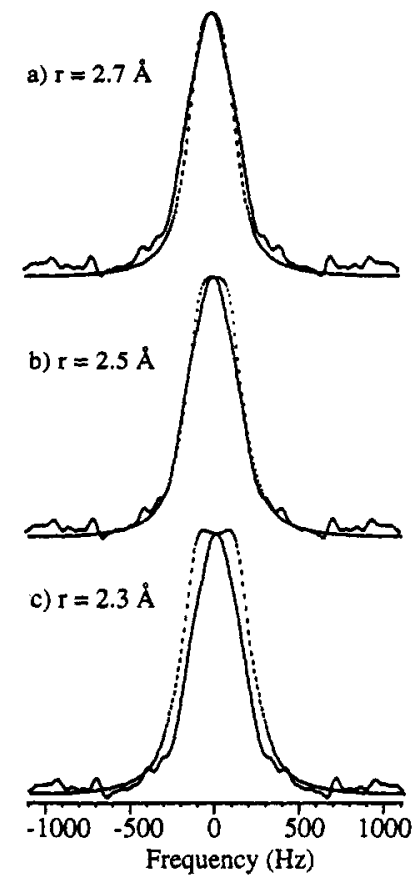

Figure 4. Slice taken from a $2 D$ spectrum of the 14-mer, H1, on the position of the carbonyl carbon in $\omega_{2}$, overlaid with simulations of broadened dipolar spectra. A total of $32 t_{1}$ slices were acquired with 1000 scans per FID and a recycle time of $1 \mathrm{~s}$. The dwell time in $t_{1}$ was $400 \mu \mathrm{s}$, and the spinning rate was $5.2 \mathrm{kHz}$. The dipolar simulations were Lorentzian broadened by $173 \mathrm{~Hz}$ and are plotted with dotted lines.

patterns. As all interactions in $\omega_{1}$ and $\omega_{2}$ are identical, with the exception of the isotropic shift in $\omega_{2}$ and the homonuclear dipolar interaction in $\omega_{1}$, the broadening function may be estimated from the MAS line width and has roughly the same influence upon fits to the data as the zero-quantum $T_{2}$ has in rotationally enhanced magnetization transfer curves. ${ }^{9}$ As an example, we present in Figure 4 slices taken from a $2 \mathrm{D}$ spectrum of a doubly ${ }^{13} \mathrm{C}$ enriched 14 amino acid peptide, $\mathrm{H} 1$, which plays a crucial role in the conformation of the prion protein (PrP) responsible for the development of scrapie in Syrian hamsters. ${ }^{20}$ The 14-mer is labeled on the C-1 and C-2 carbons of Ala-115 and Ala-116, respectively, which are connected by a peptide bond. The experimental spectra are overlaid in (a)-(c) with simulated pake patterns, broadened by the carbonyl MAS line width of $173 \mathrm{~Hz}$. The best fit to the data is spectrum $b$ with $r=2.5 \AA$. Deviations of $\pm 0.2 \AA$ from (b) yield a noticeably poorer fit. This result is in reasonable agreement with distances expected from typical peptide bond geometry, $2.45-2.49 \AA .{ }^{21}$ Future work will involve the measurement of critical distances within this and other PrP peptides, which can adopt either $\alpha$-helix or $\beta$-sheet forms. We wish to determine whether there are unusual elements in the secondary structure that could explain the pathogenicity of PrP.

\section{Experimental Section}

The doubly ${ }^{13} \mathrm{C}$ labeled zinc acetate sample was prepared by refluxing zinc oxide with $99 \%$ doubly ${ }^{13} \mathrm{C}$ labeled acetic acid (Cambridge Isotopes, Woburn, MA). The sample was recrystalized $1: 10$ in natural abundance zinc acetate to reduce intermolecular dipolar couplings, and $3 \mathrm{~mol} \%$ manganese acetate was added to shorten the proton relaxation time. All experiments were performed on a home built pulse spectrometer operating at a ${ }^{1} \mathrm{H}$ Larmour frequency of $300 \mathrm{MHz}$. The hopping probe was also home-built, ${ }^{13}$ utilized a commercial $7-\mathrm{mm}$ spinning system (Doty Scientific, Columbia, SC), and could achieve large angle reorientations of the spinning axis in approximately $50 \mathrm{~ms}$. In the fast spinning limit, the spectra were symmetric about $\omega_{1}=$ 0 ; therefore, quadrature detection in that dimension was not employed. 


\section{Conclusions}

We have described an application of the 2D dipolar SAS technique ${ }^{12}$ to the measurement of interatomic carbon-carbon distances in solids. Off magic angle spinning during the evolution period of a two-dimensional experiment, with chemical shift refocusing, has been shown to yield spectra in $\omega_{1}$ which are sensitive to the distance between labeled spins. Fits to the experimental data are sensitive only to the distance for the case of strong couplings. For instances in which the homogeneous line width is not small compared to the dipolar coupling, the fits are convolutions of the MAS line width with the idealized pake pattern. Internuclear distances thus extracted are accurate to approximately $0.2 \AA$.

Acknowledgment. This work was supported by the Director, Office of Energy Research, Office of Basic Energy Sciences, Materials Sciences Division of the U. S. Department of Energy, under Contract DE-AC03-76SF00098. M.A.B and S.B.P. acknowledge support from the National Institute of Health and the American Health Assistance Foundation. A.C.K. is a fellow of the Miller Institute for Basic Research in Science.

\section{References and Notes}

(1) Creuzet, F.; McDermott, A. E.; Gebhard, R.; vanderHoef, K.; SpijkerAssink, M.B.; Herzfeld, J,; Lugtenburg, J.; Levitt, M. H.; Griffin, R. G. Science 1991, 251, 783.

(2) Spencer, R. G. S.; Halverson, K. J.; Auger, M.; McDermott, A. E.; Griffin, R. G.; Lansbury Jr., P. T. Biochemistry 1991, 30, 10383.
(3) Pearson, O. B.; Yoshimura, S.; Hojo, H.; Aimoto, S.; Smith, S. O. J. Am. Chem. Soc. 1992, 114, 4332.

(4) Gullion, T.; Schaefer, J. Adv. Magn. Reson. 1989, 13, 57.

(5) Gullion, T.; Vega, S. Chem. Phys. Lett. 1992, 194, 423.

(6) Yannoni, C. S.; Kendrick, R. D. J. Chem. Phys. 1981, 74, 747.

(7) Horne, D.; Kendrick, R. D.; Yannoni, C. S. J. Magn. Reson. 1983, 52,299 .

(8) Raleigh, D. P.; Levitt, M. H.; Griffin, R. G. Chem. Phys. Lett. 1988, 146,71 .

(9) Levitt, M. H.; Raleigh, D. P.; Cruezet, F.; Griffin, R. G. J. Chem. Phys. 1990, 92, 6347.

(10) Bennett, A. E.; Ok, J. H.; Griffin, R. G.; Vega, S. J. Chem. Phys. $1992,96,8624$.

(11) Tycko, R.; Dabbagh, G. Chem. Phys. Lett. 1990, 173, 461.

(12) Terao, T.; Miura H.; Saika, A. J. Chem. Phys. 1986, 85, 3816.

(13) Eastman, M. A.; Grandinetti, P. J.; Lee, Y. K.; Pines, A. J. Magn. Reson. 1992, 98, 333.

(14) Tycko, R. J. Am Chem. Soc. 1994, 116, 2217.

(15) Bodenhausen, G.; Kempsell, S. P.; Freeman, R.; Hill, H. D. W. J. Magn. Reson. 1979, 35, 337.

(16) Kolbert, A. C.; Raleigh, D. P.; Levitt, M. H.; Griffin, R. G. J. Chem. Phys. 1989, 90, 679.

(17) Grandinetti, P. J.; Lee, Y. K.; Baltisberger, J. H.; Sun, B. Q.; Pines, A. J. Magn. Reson. A 1993, 102, 195.

(18) This would require a sacrifice in sensitivity, however, as the coil must be fixed, with a nonzero angle to the field, while the sample hops inside it, reducing the filling factor.

(19) Nakai, T.; McDowell, C. A. Chem. Phys. Lett. 1994, 217, 234.

(20) Gasset,M.; Baldwin, M. A.; Lloyd,D.H.; Gabriel, J.-M.; Holtzman, D. M.; Cohen,F.; Fletterick, R.; Prusiner, S. B. Proc. Natl. Acad. Sci. U.S.A. 1992, 89, 10940.

(21) Ramachandran, G. N.; Sasisekharan, V. Adv. Protein Chem. 1968, $23,284$. 